\title{
BMJ Open Fitness for purpose study of the Field Assessment Conditioning Tool (FACT): a research protocol
}

\author{
Ralph James MacKinnon, ${ }^{1}$ Chris Kennedy, ${ }^{2}$ Catherine Doherty, ${ }^{1}$ \\ Michael Shepherd, ${ }^{3}$ Joanne Cole, ${ }^{3}$ Terese Stenfors-Hayes, ${ }^{4}$ on behalf of the \\ INSPIRE Trauma Outreach Research Group (http://www.inspiresim.com)
}

To cite: MacKinnon RJ, Kennedy C, Doherty C, et al. Fitness for purpose study of the Field Assessment Conditioning Tool (FACT): a research protocol. BMJ Open 2015:5:e06386

doi:10.1136/bmjopen-2014006386

\section{- Prepublication history and additional material is available. To view please visit the journal (http://dx.doi.org/ 10.1136/bmjopen-2014- 006386).}

Received 15 August 2014 Revised 4 December 2014 Accepted 18 March 2015

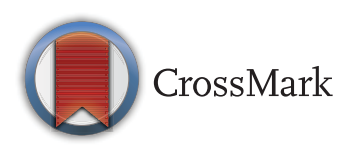

For numbered affiliations see end of article.

\section{Correspondence to}

Professor Ralph James MacKinnon;

Ralph.mackinnon@cmft. nhs.uk

\section{ABSTRACT}

Introduction: As part of a programme of research aiming to improve the outcomes of traumatically injured children, a multisource healthcare advocacy tool has been developed to allow trauma team members and hospital governance administrators to reflect and to act on complex trauma team-hospital systems interactions. We have termed this tool a Field Assessment Conditioning Tool (FACT). The FACT draws on quantitative data including clinical care points in addition to self-reflective qualitative data. The FACT is designed to provide feedback on this assessment data both horizontally across fellow potential team members and vertically to the hospital/organisation governance structure, enabling process gap identification and allowing an agenda of improvements to be realised. The aim of the study described in this paper is to explore the perceived fitness for purpose of the FACT to provide an opportunity for healthcare advocacy by healthcare professionals caring for traumatically injured children.

Methods and analysis: The FACT will be implemented and studied in three district hospitals, each around a major trauma centre in the UK, USA and New Zealand. Using a qualitative approach with standardised semistructured interviews and thematic analysis we will explore the following question: Is the FACT fit for purpose in terms of providing a framework to evaluate, reflect and act on the individual hospital's own performance (trauma team-hospital interactions) in terms of readiness to receive traumatically injured children?

Ethics and dissemination: Ethics opinion was sought for each research host organisation participating and deemed not required. The results will be disseminated to participating sites, networks and published in highimpact journals.

\section{BACKGROUND}

The rationale for this study

The aim of the study described in this paper is to explore the perceived fitness for purpose of a tool to provide an opportunity for healthcare advocacy by professionals caring for traumatically injured children.

Trauma is the leading cause of death in children less than 19 years of age in the UK and

\section{Strengths and limitations of this study}

Depth of understanding of complex relationships.

- Flexibility of approach to answering research question.

- The main disadvantage of the method of data collection is that the information provided is filtered through the respondent's memory and the social context of the interview.

worldwide. ${ }^{1}$ One of the components to improve health outcomes for injured children is a health system trained and ready to care for these children. To improve the outcomes of traumatically injured children, we have developed a multisource tool to allow trauma team members and hospital governance administrators to reflect and to act on complex trauma team-hospital systems interactions. We have termed this tool a Field Assessment Conditioning Tool (FACT) (see online supplementary appendix 1$).^{2}$ The FACT has been designed to enhance the horizontal and vertical transmission of the performance of trauma team-hospital interactions in the management of traumatically injured children. We propose that the FACT provides the opportunity to reflect on and learn from events associated with managing such children. The FACT tool has been developed to effect positive changes in staff knowledge, skills, behaviour, attitudes, team-hospital infrastructure, systems and patient care. In essence, the FACT has been designed to allow all potential members of the clinical trauma team and associated governance team to act as advocates within their own working environment to improve the care provided to traumatically injured children.

The clinical need for the FACT

Children presenting with traumatic injuries to hospitals require optimal care to decrease 
mortality and morbidity. ${ }^{3}{ }^{4}$ Optimal care provision is a complex interaction between an inter-professional team of healthcare providers, each with individual knowledge, skills and attitudes, and the healthcare system in which the team works. ${ }^{5}$ There are a number of challenges to overcome to ensure optimal care provision. One challenge is to maintain the experience of the clinicians, who may not treat children requiring major trauma care on a regular basis. ${ }^{6}$ Another challenge is to provide the opportunity for team members to reflect on the care provided in their hospital and empower them to act as advocates of change to improve care provision. ${ }^{2}$ In addition, the composition of hospital trauma teams is highly variable, with team membership changing on a daily basis and some members only staying 6 months in one hospital. ${ }^{6}$

There is a clear need for the development of an effective and systematic paediatric trauma training programme aiming to optimise the interactions between provider teams and healthcare systems in major trauma centres as well as in rural/district hospitals. ${ }^{36}{ }^{6} \mathrm{~A}$ recent study across Norway demonstrated an improvement in participant self-confidence, knowledge and perceived trauma team performance in live trauma resuscitations after initiation of an in-hospital simulation based training programme. $^{7}$ This 8-year study highlighted the potential for continued improvement in adult trauma care in those team members who had participated in the 1 day trauma training course. ${ }^{7}$

The objective of the study presented in this research protocol is to evaluate the FACT in terms of the ability of the tool:

1. To enhance the horizontal and vertical transmission of the performance of trauma team-hospital interactions in the management of injured children.

2. To provide the opportunity for all potential trauma team and governance team members to effect positive changes in staff knowledge, skills, behaviour, attitudes, team-hospital infrastructure, systems and patient care.

Recent evidence ${ }^{8-11}$ suggests that clinical care provided to children can be enhanced with targeted simulation training, using child-like human patient simulators. Highly realistic human patient simulators have been used as surrogates for real life patients in the development of the FACT and in this study to evaluate the tool.

\section{THE PRE-PILOT EVALUATION OF THE FACT}

To examine the usability, configuration and acceptability of the FACT, we conducted a small pre-pilot assessment in a major UK paediatric trauma centre. The purpose of this brief study was to evaluate whether the FACT filled a perceived gap/need for those who will receive the results. In addition, we explored whether the format is understandable and useful to identify areas of excellence and those in need of improvement for clinical, managerial and administrative staff of the hospital.
An outline of methods for pre-pilot FACT study:

1. Two onsite high-fidelity paediatric trauma scenarios were conducted at a major paediatric trauma centre in the UK.

2. A purposive sample of representative stakeholders/ providers was selected for semi-structured interviews (not involved in the scenario), including

A. Three clinicians: a senior nurse, a consultant/ attending physician from the emergency department and a neurosurgical consultant/attending.

B. Three senior trauma operations managers (including a quality manager).

3. A sample FACT report was prepared based on the results from the two scenarios and presented $48 \mathrm{~h}$ prior to the interview process.

4. Semi-structured interviews were conducted by one of the investigators (RJM), the interviews were recorded and transcribed verbatim.

5. The transcripts were analysed for common themes related to whether the format was usable, the transcripts understandable, and their potential uses.

6. A brief summary of findings was prepared.

The interviews initially focused on the respondents' understanding of quality outcomes in the trauma setting, and their own role in improvement work. The second part of the interviews focused on the configuration, reliability, validity, educational impact and acceptability of the FACT, to provide an opportunity for healthcare advocacy.

Analysis of the first part of the interviews revealed a common difficulty in measuring quality of care. No tool at the time was identified to facilitate reflection and learning on high quality care provision occurring at the pre-pilot centre. An audit process focusing on reaching target times was identified and high-risk poor clinical management reviewed by a root cause analysis mechanism. However, no individual/team based advocacy opportunities were identified. Respondents felt a need for a balanced input in terms of external and internal expertise to assess performance, but lacked a tool for this process. This pre-pilot thereby supports the underlying concepts of developing a tool such as the FACT.

The second part of the interviews revealed a uniform sense that the FACT does offer a common language to provide the opportunity to invoke healthcare advocacy changes, and that it is acceptable in the current configuration and format. It was also stated that evolution to an online version could be desirable.

\section{THIS STUDY: A FITNESS FOR PURPOSE STUDY OF THE FACT}

\section{Study aim}

The aim of the project is to explore the perceived fitness for purpose of the FACT to provide an opportunity for healthcare advocacy by healthcare professionals caring for traumatically injured children. We use the term 'fitness for purpose' here as a criterion for establishing whether or not a tool provides quality, measured against 
what is seen to be the goal of the tool. In this context, we define healthcare advocacy as the empowerment of all potential trauma team members to make changes to improve their own team and hospital system interactions during the management of traumatically injured children.

To achieve this aim, a qualitative approach was deemed most suitable. To explore the fitness for purpose of the FACT, we have considered the work of Van der Vleuten, ${ }^{12}$ who previously described a 'utility index' as a framework for tool design and evaluation. The utility index has five components: reliability, validity, educational impact, cost-efficiency and acceptability. At this stage we are not in a position to assess the costefficiency of the introduction of the FACT, however, the other components will be explored to gain information that will highlight areas in need of development in the FACT. By conducting interviews we gain access to the respondent's personal perspectives, and experiences of the FACT process and results. The main disadvantage of this method of data collection is that the information provided is filtered through the respondent's memory and the social context of the interview. ${ }^{13}$

\section{METHODOLOGY}

\section{Ethical issues}

Ethics opinion has been sought from research host organisations. In the UK, in accordance with current governance guidelines for health research, studies on NHS staff and service provision, and the engagement of the general public to seek opinion of this service provision, this research project does not require NHS Research Ethics Approval. All participating individuals consent for audiovisual recording as part of this study and are provided with a participant information document. In New Zealand, the research team has received approval through the regional research committee and ethics waiver through the National Health and Disability Ethics committee. Consent will be sought and provided by all participants for audiovisual recording. In the USA, ethics approval has been provided by Children's Mercy Hospital Pediatric Institutional Review Board, Kansas City. All participants will be consented for audiovisual recording of their participation in the study.

\section{Study design}

Using a qualitative approach with standardised semi-structure interviews and thematic analysis, we will explore the following question: Is the FACT fit for purpose in terms of providing a framework to evaluate, reflect and act on the individual hospital's own performance (trauma team-hospital interactions) in terms of the readiness to receive traumatically injured children?

\section{Study timeline}

The study is being conducted in the UK, USA and New Zealand, at three major paediatric trauma centres. The study will be conducted over a 1-year period, as depicted in table 1. A FACT report will be constructed at each participating hospital, as detailed in online supplementary appendix 1. The FACT will be disseminated to all potential trauma team members. This process will be repeated at 6 months and then semi-structured interviews will be conducted at each of the participating hospitals, to assess the fitness for purpose of the FACT.

\section{Sample and recruitment}

The FACT tool will be implemented according to online supplementary table 2 in appendix 1, within three district/non-paediatric hospitals around each participating major trauma centre in the study. The principle investigator at each major trauma centre will recruit the district/non-paediatric hospitals and consent participating individuals at each hospital. At time of manuscript submission, UK and New Zealand major trauma centres had recruited three district/non-paediatric hospitals.

\section{Data collection}

Semi-structured interviews will be conducted at each of the participating hospitals, using a purposeful sample to achieve maximum variation. The interview is guided by a pre-determined set of open-ended questions (see online supplementary appendix 2). However, the researcher and respondents are free to pursue additional topics that are deemed relevant. ${ }^{14}$ We aim to sample potential clinical interprofessional members of trauma teams and also hospital administrators/managers. A minimum of 12 interviews will be conducted at each hospital with representative variation in the respondents' profession, years in practice, gender and age. Variation in the sample will enable a broad range of perspectives and experiences to be captured, and thereby lead to a deepened understanding. Previous research has shown that saturation of findings occurs within the first 12 interviews, even when the variation in the sample is high (such as different national contexts). ${ }^{15}$ Owing to the high variation within each national context in our study, we have chosen a minimum of 12 respondents at each site. Participants will be recruited via an email that provides information about the study and invites participation. The interviews will be conducted within 2 weeks of the FACT result from the second pilot test being made available to the hospital. The semi-structured interviews will be conducted by an external research team member not known to the respondent, and who has not been part of developing the FACT tool to avoid bias and power dynamics in the interview situation. The interviews will be conducted at a time and place convenient for the participant. All interviews will be audio or video recorded and transcribed. The findings of prepilot interviews were used to formulate the interview guide with all participants encouraged to freely express their views, with the expectation of no correct or incorrect answers and all viewpoints accepted. 


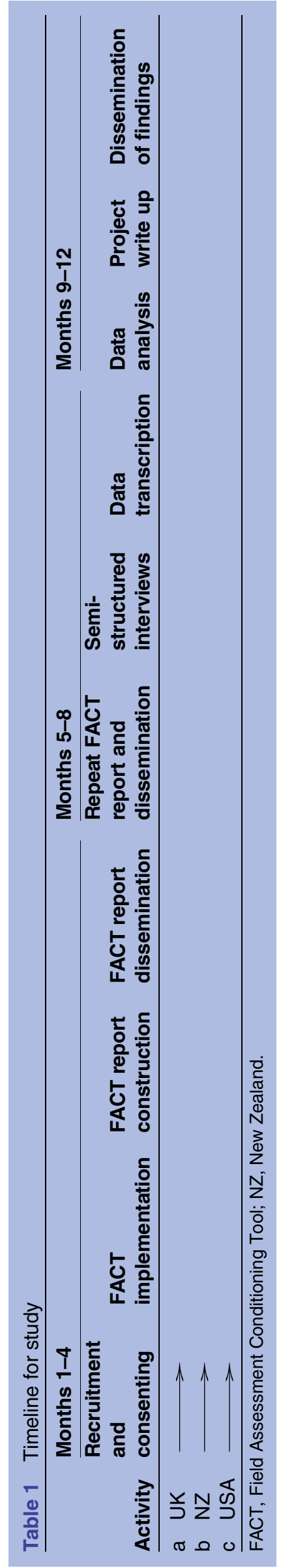

Data analysis

All interviews will be analysed using an inductive thematic approach within the broad framework of the utility index. A thematic approach has the advantage that it can be applied across a range of theoretical and epistemological approaches. Through its theoretical freedom, thematic analysis provides a flexible and useful research tool that can potentially provide a rich and detailed, yet complex account of data. ${ }^{16}$ An inductive approach means the themes identified are strongly linked to the data themselves. ${ }^{17}$ Inductive analysis is therefore a process of coding the data without trying to fit it into a pre-existing coding frame, or the researcher's analytic preconceptions. The analytic process will include interpretation at the latent level, which goes beyond the semantic content of the data, and starts to identify or examine the underlying ideas, assumptions and conceptualisations. However, as we have chosen to relate our findings to the utility index, our analysis will include some deductive aspects as well as the inductive.

The interviewer (an external $\mathrm{PhD}$ trained qualitative researcher) and one local research team member will analyse the data from each centre independently before they meet and review the coding, until consensus is reached. The local team member will be able to add to the analysis by their fuller contextual understanding and medical knowledge, while the external researcher will reduce bias, as they have not been involved in the development of the tool and not present when the FACT was tested. When reading the transcripts, ideas expressed by the informants will be condensed, compared and grouped into themes that represent similar ways of understanding the phenomena under scrutiny. This process will occur iteratively, that is, as new perspectives arise they will be examined in the context of the entire data set. The analytic process used is similar to what is commonly referred to as constant comparison. ${ }^{18}$ The themes are not dependent on quantifiable measures, but in terms of whether they capture something important in relation to the overall research question. Once data from all three centres are analysed, the findings will be compared and contrasted. Maintaining an audit trail of the interview transcripts, analytical memos and the developing themes and their relations will ensure dependability of the data. ${ }^{19}$ The trustworthiness of the findings will be strengthened because the analysis is being conducted by a team of researchers who will discuss and debate their interpretations until consensus is achieved. ${ }^{20}$

\section{Strengths and limitations}

The strengths of this study include the depth of understanding of complex relationships that will be explored and the flexibility of the methodological approach to facilitate answering of the research question. The main disadvantage of the method of data collection is that the information provided is filtered through the respondent's memory and the social context of the interview. ${ }^{13}$ 


\section{Dissemination of findings}

The results of this study will be disseminated to participating sites, regional and international trauma networks and submitted to high-impact journals.

\section{DISCUSSION}

The aim of the study described in this paper is to explore the perceived fitness for purpose of the FACT to provide an opportunity for healthcare advocacy by professionals caring for traumatically injured children. The fitness for purpose of the FACT, in terms of configuration, reliability, validity, educational impact and acceptability will be qualitatively analysed. This analysis will provide an invaluable insight into the development of a tool to promote patient safety and improved quality of care for high-risk complex children who present infrequently to hospitals. This study will also shed further light on our understanding of the measurement of quality in the paediatric trauma setting and how we collect, represent and display key learning opportunities across an ever changing mix of staff.

Recent discussion on the epistemology of patient safety (defined as the science of the method of finding about patient safety) from the perspective of a risk management framework, has developed key issues in this domain. ${ }^{21}$ These include identifying, analysing, evaluating and managing risk. ${ }^{21}$ The authors also emphasise the importance of a deep understanding of the context where healthcare delivery occurs, the need for communication, monitoring and review. ${ }^{21}$ With the goal of patient safety at the forefront, the authors highlight the strength of combining a mixed qualitative and quantitative framework approach to achieve this. We propose that the FACT uses this mixed approach and builds on these patient safety principles, particularly with reference to the $\delta$ elements of the tool. We aim to also highlight excellent care provision by paediatric trauma teams (the positive elements of the FACT), and disseminate this horizontally and vertically, as discussed above, to all potential team members and advocates, to improve care.

The development of a tool that provides a common language, horizontally across team-members and vertically through the governance tree, and also empowers individuals throughout an organisation to invoke positive changes, has the potential to improve patient care. The input from a parental perspective will constitute a different but equally vital addition to this process. At this stage, we will only be exploring the perception of staff throughout the hospital organisations as to whether they have been provided with the opportunity to change staff behaviour, impact positively on trauma education, change hospital systems, reduce adverse events and improve patient care. The next step will be to optimise the FACT as a healthcare advocacy tool as per our findings from this study. With an optimal FACT in operation in hospitals, we aim as a research group to introduce trauma care interventions that we have developed, to directly impact positively on care provision.
While the primary focus of this project is to evaluate a healthcare advocacy tool to empower potential trauma team members to make changes to improve their own team and hospital system interactions, in forthcoming research we also intend to explore whether other educational interventions have the potential to positively effect 'system change' and directly impact patient care. This is a novel application of in-situ simulation, with the use of simulation-based education directly at the place of work where healthcare professionals provide care. This method of assessment may be cutting edge, as traditional methods that are typically used to change systems include policy, regulatory and political interventions. We must be cognisant that effecting system change may not occur as a result of this approach, however, one can visualise that local healthcare systems will benefit from this operationalised approach and the summary recommendations that will result.

\section{Author affiliations}

${ }^{1}$ Department of Paediatric Anaesthesia, Royal Manchester Children's Hospital, Manchester, UK

${ }^{2}$ Emergency \& Urgent Care, Children's Mercy Hospital, Kansas City, Kansas, USA

${ }^{3}$ Children's Emergency Department, Starship Children's Hospital, Auckland, New Zealand

${ }^{4}$ Department of Learning, Informatics, Management and Ethics, Karolinska Institutet, Stockholm, Sweden

Collaborators The INSPIRE Trauma Outreach Research Group includes RJM, CK, Dr Marc Auerbach, Dr Mary Patterson, Dr David Kessler, Dr Betsy Hunt, Dr Vince Grant, Dr Corsino Rey, Dr Jesus Lopez-Herce, Dr Antonino Rodriguez-Nunez, Dr Lambert Schuwirth, MS, Dr Todd Chang, Dr Phil Hyde, TS-H and Dr Vinay Nadkarni.

Contributors All authors have provided substantial contributions to the conception or design of the work, given approval for publishing and are accountable for all aspects of the work.RJM and CK provided the initial concept. TS-H added qualitative analysis concepts. MS, CD and JC working with RJM, CK and TS-H developed the design of the protocol together, adding elements relating to country-specific healthcare systems and wider design points. All team members have communicated closely on this protocol, prior to this submission.

Funding This project has been awarded small grant funding from Health Education-North West (Greater Manchester) UK and the Auckland District Health Board Charitable Trust, New Zealand.

Competing interests None.

Ethics approval Central Manchester Foundation Trust.

Provenance and peer review Not commissioned; externally peer reviewed.

Open Access This is an Open Access article distributed in accordance with the Creative Commons Attribution Non Commercial (CC BY-NC 4.0) license, which permits others to distribute, remix, adapt, build upon this work noncommercially, and license their derivative works on different terms, provided the original work is properly cited and the use is non-commercial. See: http:// creativecommons.org/licenses/by-nc/4.0/

\section{REFERENCES}

1. Department of Health. Saving lives: our healthier nation. London: Stationery Office, 1999. https://www.gov.uk/government/uploads/ system/uploads/attachment_data/file/265576/4386.pdf (accessed 31 Sep 2014). 
2. Kennedy C, Schuwirth L, Fleming R, et al. Development of a Field Assessment Conditioning Tool (FACT) -an exploration of the role of healthcare advocacy 2014, in press.

3. Esposito TJ, Sanddal ND, Hansen JD, et al. Analysis of preventable trauma deaths and inappropriate trauma care in rural state. J Trauma 1995;39:955-62.

4. Esposito T, Sanddal T, Reynolds S, et al. Effect of a voluntary trauma system on preventable death and inappropriate care in a rural state. J Trauma 2003;54:663-9.

5. Hjortdahl M, Ringen $\mathrm{AH}$, Naess $\mathrm{AC}$, et al. Leadership is the essential non-technical skill in the trauma team-results of a qualitative study. Scand J Trauma Resusc Emerg Med 2009;17:48

6. Trauma who cares? A report of the National Confidential Enquiry into Patient Outcome and Death. 2007. http://www.ncepod.org.uk/ 2007report2/Downloads/SIP_report.pdf (accessed 22 Sep 2014).

7. Wisborg T, Brattebø G, Brinchmann-Hansen A, et al. Effects of nationwide training of multiprofessional trauma teams in Norwegian hospitals. J Trauma 2008;64:1613-18.

8. Neily J, Mills $\mathrm{P}$, Young-Xu, Y, et al. Association between implementation of a medical team training program and surgical mortality. JAMA 2010;304:1693-700.

9. Friedman Z, You-Ten K, Bould M, et al. Teaching lifesaving procedures: the impact of model fidelity on acquisition and transfer of cricothyrotomy skills to performance on cadavers. Anesth Analg 2008;107:1663-9.

10. Wheeler DS, Geis G, Mack EH, et al. High-reliability emergency response teams in the hospital: improving quality and safety using in situ simulation training. BMJ Qual Saf 2013;22:507-14.
11. Gardner R, Walzer A, Simon R, et al. Obstetric simulation as a risk control strategy: course design and evaluation. Simul Healthc 2008;3:119-27.

12. Van Der Vleuten CP. The assessment of professional competence: developments, research and practical implications. Adv Health $\mathrm{Sci}$ Educ Theory Pract 1996;1:41-67.

13. Reeves S, Lewin S, Zwarenstein M. Using qualitative interviews within medical education research: why we must raise the 'quality bar'. Med Educ 2006;40:291-2.

14. DiCicco-Bloom B, Crabtree BF. The qualitative research interview. Med Educ 2006;40:314-21

15. Guest G, Bunce A, Johnson L. How many interviews are enough? An experiment with data saturation and variability. Field Methods 2006;18:59.

16. Braun V, Clarke V. Using thematic analysis in psychology. Qual Res Psychol 2006;2:77-101.

17. Patton MQ. Qualitative evaluation and research methods. 2nd edn Newbury Park, CA: Sage Publications, Inc., 1990.

18. Strauss A, Corbin JM. Basics of qualitative research: grounded theory procedures and techniques. Thousand Oaks, CA, USA: Sage Publications, Inc., 1990.

19. Bryman A. The nature of qualitative research. In: Bryman A, ed. Social research methods. 3rd edn. Oxford: Oxford University Press, 2008:366-99.

20. Stenfors-Hayes T, Hult H, Dahlgren MA. A phenomenographic approach to research in medical education. Med Educ 2013;47:261-70

21. Runciman WB, Baker GR, Michel P, et al. The epistemology of patient safety research. Int J Evid Based Healthc 2008;4:476-86. 